\title{
Role of amino acids in rheumatoid arthritis studied by metabolomics
}

\author{
Min HE,${ }^{1,2}$ Amy C. HARMS, ${ }^{1}$ Eduard VAN WIJK, ${ }^{1,2}$ Mei WANG,${ }^{1,2,3}$ Ruud BERGER, ${ }^{1}$ \\ Slavik KOVAL, ${ }^{1}$ Thomas HANKEMEIER ${ }^{1,2}$ and Jan VAN DER GREEF ${ }^{1,2,4}$ \\ ${ }^{1}$ Analytical BioSciences, LACDR, Leiden University, ${ }^{2}$ Sino-Dutch Center for Preventive and Personalized Medicine, Leiden \\ University, Leiden, ${ }^{3} \mathrm{~S} U$ Biomedicine, and ${ }^{4} \mathrm{TNO}$, Zeist, the Netherlands
}

\begin{abstract}
Background: Rheumatoid arthritis (RA) is a complex, chronic autoimmune disease characterized by various inflammatory symptoms, including joint swelling, joint pain, and both structural and functional joint damage. The most commonly used animal model for studying RA is mice with collagen-induced arthritis (CIA); the wide use of this model is due primarily to many similarities with RA in human patients. Metabolomics is used increasingly in biological studies for diagnosing disease and for predicting and evaluating drug interventions, as a large number of disease-associated metabolites can be analyzed and interpreted from a biological perspective.

Aim: To profile free amino acids and their biogenic metabolites in CIA mice plasma.

Method: Ultra-high-performance liquid chromatography/tandem mass spectrometry coupled with multiple reaction monitoring (MRM) was used for metabolomics study.

Results: Profile of 45 amine metabolites, including free amino acids and their biogenic metabolites in plasma was obtained from CIA mice. We found that the plasma levels of 20 amine metabolites were significantly decreased in the CIA group.

Conclusion: The results suggest that a disordered amine response is linked to RA-associated muscle wasting and energy expenditure.
\end{abstract}

Key words: amine metabolites, collagen-induced arthritis, mouse model, systems biology.

\section{INTRODUCTION}

Rheumatoid arthritis (RA) is a highly prevalent chronic disease, currently affecting approximately $1 \%$ of the world's population. ${ }^{1-3}$ Patients with RA typically have destruction of joint cartilage and bone accompanied by joint stiffness, hyperplasia, microvascular injury, swelling and pain. The pathogenesis of RA is mainly associated with the secretion of cytokines such as interleukins (e.g., IL-1 and IL-6), tumor necrosis factor (TNF $\alpha$ ), interferon gamma (IFN $\gamma)$, and various pro-

Correspondence: Dr. Eduard van Wijk, Division of Analytical Biosciences, LACDR, Sino-Dutch Centre for Preventive and Personalized Medicine, Leiden University, 2333 CC Leiden, The Netherlands. Email: eduard.vanwijk@sinodutchcentre.nl inflammatory mediators. ${ }^{4,5}$ Increased activity of the nuclear factor (NF)- $\mathrm{KB}$ pathway, which inhibits apoptosis in immune cells, also plays a role in RA. ${ }^{6-10}$ A variety of cellular immune responses are also activated and/or dysregulated by increased cytokine levels in RA. ${ }^{11-14}$ Interestingly, nearly two-thirds of patients with RA develop cachexia and sarcopenia, with a loss of skeletal muscle mass, degradation of proteins and energy expenditure. ${ }^{15-18}$ This perturbation in catabolic processes drives the body into a state of negative energy balance, leading to skeletal muscle atrophy, loss of muscle strength and reduced physical activity. ${ }^{18,19}$

Considering the complex nature of RA, animal models have been useful for studying the underlying pathology and disease mechanisms. The most widely used 
animal model for studying chronic RA is the collageninduced arthritis (CIA) mouse model; in addition to high reproducibility and easy induction, the physiological processes and pathogenic features of CIA mice are strikingly similar to the clinical features associated with patients with RA. ${ }^{20-23}$ For example, increased levels of IL-6, IL-1, and TNF $\alpha$ play a role in the development of CIA. $^{24}$ In addition, high correlation between muscle wasting and the severity of clinical arthritis has also been observed in animal models, including both monkeys and mice with CIA. ${ }^{25,26}$

Applying a systems biology approach using metabolomics can provide a comprehensive functional readout of the organism's physiological status. ${ }^{27}$ Recently, van Wietmarschen and van der Greef summarized the putative inflammatory mediators identified in RA patients using metabolomics. ${ }^{28}$ Although some pro-inflammatory mediators have been observed in CIA mice, ${ }^{29}$ the complexity of the disease warrants a search for additional compound classes and a study of their relationship with the biochemical processes underlying RA. Free amino acids and their derivative biogenic amines play essential roles in both energy production and protein synthesis/degradation; thus, changes in the levels of these amine metabolites may reflect changes in the body's state and catabolism of proteins in RA disease. Therefore, we used a liquid chromatography mass spectrometry (LC/MS)-based amine platform to measure the levels of amine metabolites in the plasma of CIA and control mice. We observed reduced levels of amine metabolites in the plasma of CIA mice, possibly reflecting systemic changes in this model of RA. Based on these results, we speculate that decreased amine metabolite levels likely reflect muscle mass loss and protein degradation and may associate with inflammatory activity.

\section{MATERIALS AND METHODS}

\section{Induction of arthritis by co-administration of collagen type II and lipopolysaccharide}

A total of 20 male DBA/1J mice (age 6-7 weeks) were obtained from Charles River Laboratories (Yokohama, Japan). The animals were randomly divided into two groups, with 10 mice in the experimental (CIA) group and 10 mice in the control (Ctrl) group. The protocol for inducing arthritis is well established and has been described in detail. ${ }^{29-31}$ In brief, the mice were given intraperitoneal (i.p.) injections containing collagen type II (extracted from bovine nasal cartilage and dissolved in acetic acid) and lipopolysaccharide (extracted from Escherichia coli 011:B4 and dissolved in phosphate-buffered saline) in order to induce chronic polyarthritis by stimulating an autoimmune response; control mice received i.p. injections of vehicle (acetic acid and phosphate-buffered saline) only. All animals were housed in a temperature- and lightcontrolled environment with free access to standard rodent chow and water throughout the experiments. After repeated injections (administered on days 0, 14, 28,42 and 56), blood samples were collected from each animal on day 70 and stored in pre-cooled Vacutainer tubes (BD Vacutainer, Plymouth, UK) containing ethylenediaminetetraacetic acid (EDTA) as an anticoagulant. After centrifugation, the EDTA-plasma fractions were collected and aliquots, including individual study samples and pooled quality control (QC) samples, were stored at $-80^{\circ} \mathrm{C}$ until further analysis. During sampling, one mouse in the CIA group died; thus, the final analysis is based on nine CIA mice and 10 control mice.

\section{Extraction of amine metabolites and analysis using UPLC-MS/MS}

The methods for extracting and analyzing amine metabolites were adapted for mouse plasma samples based on a previously described protocol. ${ }^{32}$ For each sample, a $5-\mu \mathrm{L}$ aliquot of plasma was used for the analysis. A mixture of internal standards containing ${ }^{13} \mathrm{C}^{15} \mathrm{~N}$ labeled amine metabolites was added to each $5-\mu \mathrm{L}$ plasma sample. After the proteins were precipitated using $\mathrm{MeOH}$, the supernatant was transferred to a fresh Eppendorf tube and dried under $\mathrm{N}_{2}$. The residue was then dissolved in borate buffer ( $\mathrm{pH} 9)$, and 6-aminoquinolyl-N-hydroxysccinimidyl carbamate (AQC) derivatization reagent (Waters, Etten-Leur, The Netherlands) was added. The reaction mixture was then neutralized by the addition of formic acid (20\%), and the solution was transferred to injection vials for ultrahigh-performance LC tandem MS (UPLC-MS/MS) analysis (injection volume: $1.0 \mu \mathrm{L}$ ) using an ACQUITY UPLC system (Waters) equipped with an AccQ-Tag Ultra column $(2.1 \mathrm{~mm} \times 100 \mathrm{~mm}, 1.7 \mu \mathrm{m}$ particles, Waters) coupled to a Xevo mass spectrometer with electrospray ionization source (Waters). Multiple reaction monitoring was performed in the positive ion mode in order to monitor the analytes. A gradient elution starting with Eluent $\mathrm{A}$ (water containing $2 \%$ formic acid) and ramping to Eluent B (aqueous acetonitrile containing $2 \%$ formic acid) was used as the mobile phase in the UPLC system. The samples were analyzed in random order. 


\section{Data processing and statistical analysis}

The integrated peak areas of the target analytes were calculated using Quanlynx software (Waters) and corrected using the appropriate internal standards. The response ratio (calculated at the ratio between the target analyte and the respective internal standard) was used for further statistical analysis. The reproducibility and reliability of each metabolite measurement was determined using repeated measurements of the QC pool performed after every 10 samples. By defining the acceptable relative standard deviation as $<15 \%, 45$ amine metabolites (from a starting list of 74) were considered high quality and were included in the final list for further analysis. The data were log-transformed to correct for distribution skewness and auto-scaled to achieve uniform units.

To visualize clustering of individual samples, unsupervised principal component analysis (PCA) was performed using MetaboAnalyst version 3.0 (http://www. metaboanalyst.ca). ${ }^{33}$ To measure the significance of differences in each individual amine metabolite between the CIA group and the Ctrl group, a two-sided unpaired Student's $t$-test was performed, assuming unequal variance; differences with a $P$-value $<0.05$ were considered significant $\left(\mathrm{H}_{0}\right.$ : group means are equal). Fold change (FC) was then calculated in order to determine the direction ( $\log _{2}$ of FC) and magnitude (FC ratio reflecting the CIA/Ctrl ratio) of differences between two group mean values. A positive value for the $\log _{2}$ of FC indicates higher levels of metabolites in the CIA group, whereas a negative value indicates lower levels of metabolites in the CIA group. In the FC analysis, a minimum threshold of 1.5 was used, meaning that the ratio of metabolites between the CIA and Ctrl groups exceeded 1.5.

\section{RESULTS}

\section{Univariate and multivariate analyses}

A 2D plot of the PCA scores was generated using an unsupervised pattern recognition method and was used to provide a visual overview of the natural distribution of amines detected in the plasma samples of the nine CIA and 10 Ctrl mice (Fig. 1). PC1 and PC2 accounted for $56.6 \%$ and $13.9 \%$ of the variation, respectively; thus, these two principal components (i.e., PC1 and PC2) explained a total of $70.5 \%$ of the variance. From the 2D plot of the PCA scores, the CIA group (depicted with triangle symbols) and the Ctrl group (depicted with the ' + ' symbol) were generally distributed in

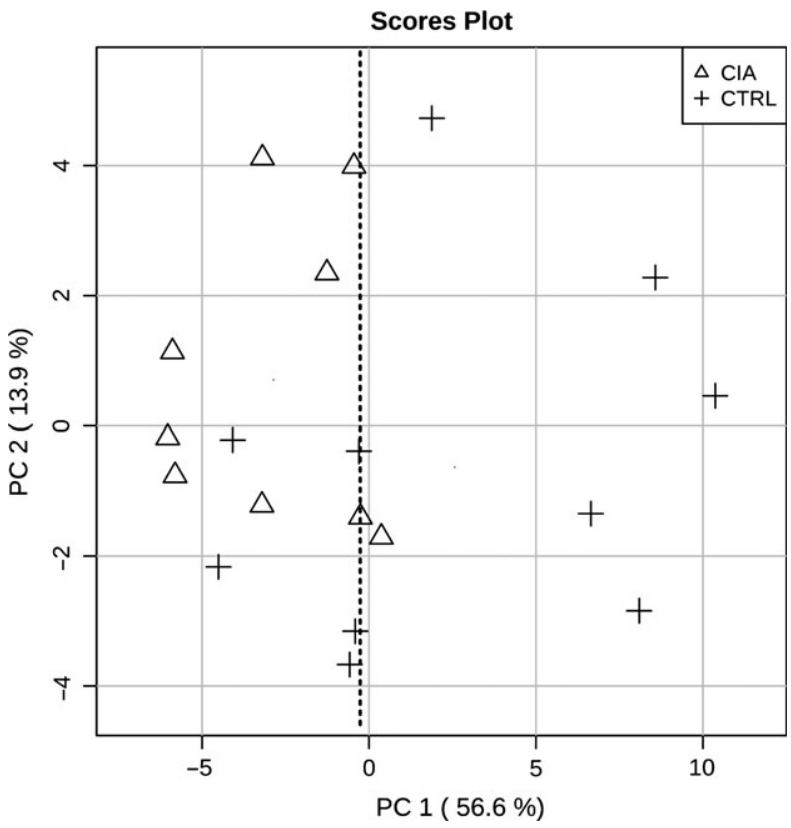

Figure 1 2D plot of the principal components analysis (PCA) scores for the amine metabolites measured in the plasma samples from collagen-induced arthritis (CIA) $(\triangle)$ and control $(+)$ mice. The plot of the PCA scores shows that the two groups form distinct clusters along the $x$-axis (corresponding to PC1), indicated by the vertical dashed line.

distinct regions with respect to PC1, with the CIA samples clustering largely on the negative side of the plot and the Ctrl samples clustering largely on the positive side of the plot, thereby reflecting group differences with respect to the composition of free amine metabolites in the plasma samples.

To measure whether the differences between the two groups were significant, we performed a Student's $t$-test for each amine metabolite. In total, 20 of the 45 detected amine metabolites differed significantly between the two groups $(P<0.05)$; these 20 amine metabolites are shown in Figure 2, and all 45 detected amine metabolites are summarized in Table 1. Metabolite changes are reported in Table 1 only when the $P$-values from the Student's test were lower than 0.1 . Table 1 also lists the false discovery rate-adjusted $P$-values. FC analysis was performed to indicate the direction of change and the magnitude of change for the detected amine metabolites (FC of the CIA/Ctrl ratio). The analysis revealed that 11 amine metabolites decreased by more than one-third in the CIA group $\left(\mathrm{FC}_{\mathrm{CIA} / \mathrm{Ctrl}}<0.67\right)$. The $\log _{2}$ value of $\mathrm{FC}$ indicates that 43 of the 45 amine metabolites detected $(95.6 \%)$ were 
Figure 2 Summary of the 20 amine metabolites that differed significantly between the collagen-induced arthritis (CIA) and control groups $(P<0.05)$. The values are presented as the response ratio of the peak area (determined as the ratio of the target amine metabolite to its corresponding internal standard) after logarithmic transformation and auto-scaling.

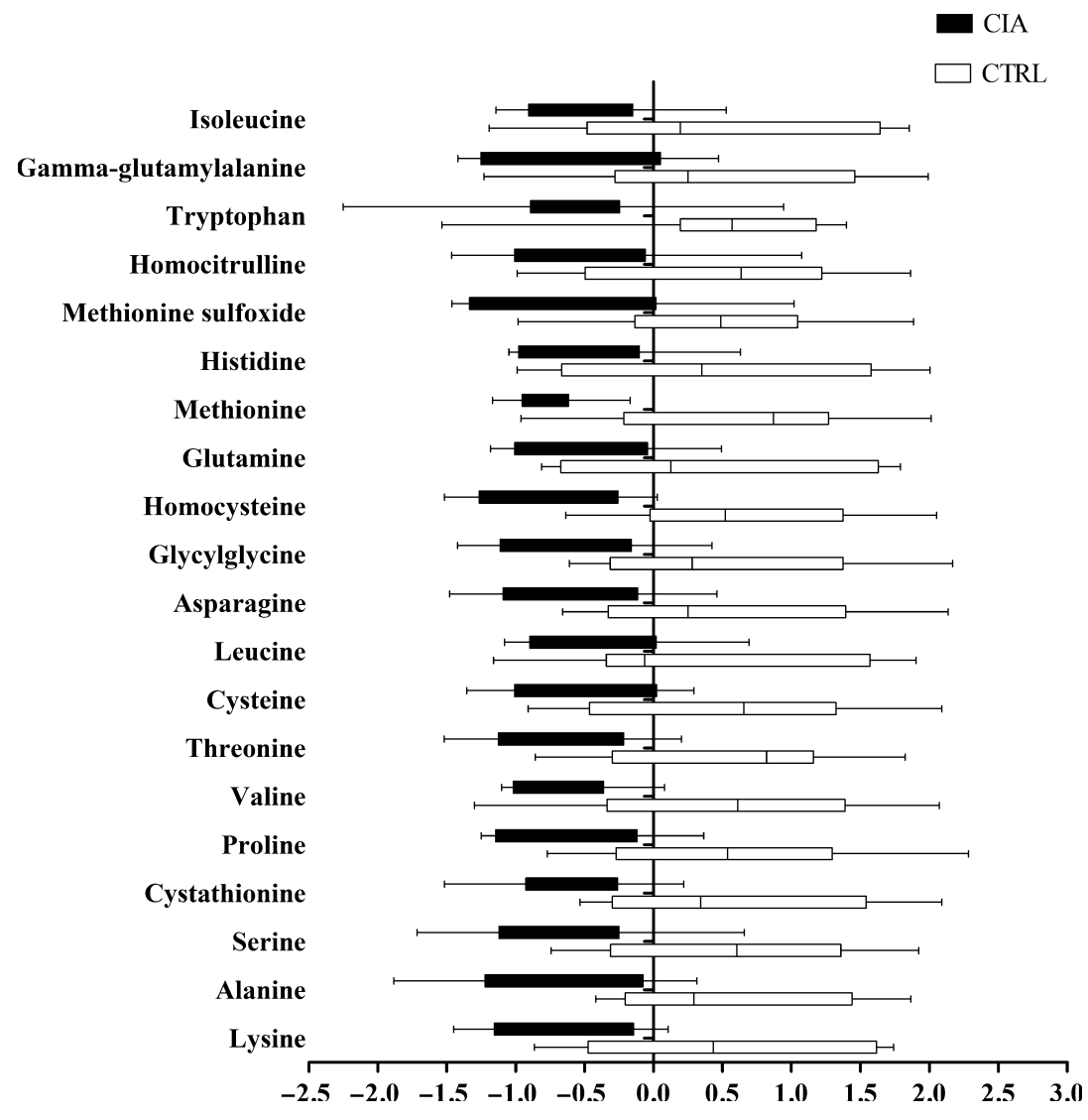

lower in the CIA group (i.e., a negative $\log _{2}$ value of FC), whereas the remaining two metabolites (methylcysteine and O-phosphoethanolamine) were higher in the CIA group.

\section{DISCUSSION}

RA is a chronic disease in which the immune response is dysregulated and the levels of several cytokines and factors are elevated, including TNF $\alpha$, IL-1 $\beta$, IL-6, IFN $\gamma$, and reactive oxygen species (ROS) ${ }^{34,35}$ in addition, NF$\kappa \mathrm{B}$ activation is increased. ${ }^{36}$ Changes in metabolic factors such as arachidonic acid-derived inflammatory mediators have also been reported in RA, ${ }^{37}$ suggesting that a metabolomics approach may provide insight into the biochemical processes underlying this disease.

In addition to the well-characterized inflammatory dysregulation in RA, muscle wasting and energy expenditure are also common features and are linked to the production of cytokines during the immune response; $;^{38-40}$ muscle wasting and energy expenditure can then dysregulate the protein degradation pathway, leading to perturbed metabolic processes. ${ }^{15,16,19,41-43}$ Given the close relation between amine metabolites and proteins, it is therefore reasonable to speculate that changes in amine metabolites may reflect protein dysregulation leading to muscle wasting and energy expenditure. However, few studies have focused on measuring muscle wasting in RA by measuring the plasma levels of amine metabolites.

Studies of the biochemical processes associated with RA revealed that activated NF- $\kappa \mathrm{B}$ is linked to skeletal muscle loss, ${ }^{44}$ and this activation has been observed in animal models of RA. ${ }^{45,46}$ Moreover, injecting TNF and IL-1 into healthy rats causes muscle wasting. ${ }^{47}$ Previously, we reported increased levels of inflammatory mediators and ROS-generated oxylipins in the plasma of CIA mice, and this was associated with the production of cytokines and increased NF- $\kappa$ B activation. ${ }^{29}$ Increased ROS levels, which affect muscle signaling pathways, have also been measured in CIA mice; ${ }^{48}$ similar results have been reported in tumor-bearing rats. ${ }^{49}$ Given that increased cytokines, ROS and NF- $\kappa$ B activation robustly affect muscle metabolism, we expected to 


\begin{tabular}{|c|c|c|c|c|c|}
\hline Amine Mmetabolite & HMDB & $P$-value & FDR & $\mathrm{FC}_{\mathrm{CIA} / \mathrm{Ctrl}}$ & $\begin{array}{l}\text { Direction } \\
\text { of change }\end{array}$ \\
\hline Methionine & HMDB00696 & 0.001 & 0.017 & 0.533 & $\downarrow$ \\
\hline Homocysteine & HMDB00742 & 0.001 & 0.017 & 0.640 & $\downarrow$ \\
\hline Threonine & HMDB00167 & 0.001 & 0.017 & 0.628 & $\downarrow$ \\
\hline Proline & HMDB00162 & 0.003 & 0.031 & 0.521 & $\downarrow$ \\
\hline Alanine & HMDB00161 & 0.003 & 0.031 & 0.573 & $\downarrow$ \\
\hline Cystathionine & HMDB00099 & 0.005 & 0.032 & 0.818 & $\downarrow$ \\
\hline Valine & HMDB00883 & 0.005 & 0.032 & 0.650 & $\downarrow$ \\
\hline Glycylglycine & HMDB11733 & 0.006 & 0.032 & 0.660 & $\downarrow$ \\
\hline Lysine & HMDB00182 & 0.007 & 0.032 & 0.686 & $\downarrow$ \\
\hline Serine & HMDB00187 & 0.007 & 0.032 & 0.815 & $\downarrow$ \\
\hline Asparagine & HMDB00168 & 0.009 & 0.036 & 0.667 & $\downarrow$ \\
\hline Cysteine & HMDB00574 & 0.012 & 0.044 & 0.802 & $\downarrow$ \\
\hline Tryptophan & HMDB00929 & 0.015 & 0.051 & 0.789 & $\downarrow$ \\
\hline Homocitrulline & HMDB00679 & 0.017 & 0.051 & 0.737 & $\downarrow$ \\
\hline $\begin{array}{l}\text { Methionine } \\
\text { sulfoxide }\end{array}$ & HMDB02005 & 0.017 & 0.051 & 0.582 & $\downarrow$ \\
\hline Isoleucine & HMDB00172 & 0.020 & 0.056 & 0.682 & $\downarrow$ \\
\hline $\begin{array}{l}\text { Gamma- } \\
\text { glutamylalanine }\end{array}$ & HMDB06248 & 0.021 & 0.056 & 0.616 & $\downarrow$ \\
\hline Histidine & HMDB00177 & 0.041 & 0.103 & 0.799 & $\downarrow$ \\
\hline Glutamine & HMDB00641 & 0.047 & 0.107 & 0.775 & $\downarrow$ \\
\hline Leucine & HMDB00687 & 0.048 & 0.107 & 0.741 & $\downarrow$ \\
\hline Citrulline & HMDB00904 & 0.052 & 0.112 & 0.818 & $\downarrow$ \\
\hline Saccharopine & HMDB00279 & 0.086 & 0.168 & 0.698 & $\downarrow$ \\
\hline Ornithine & HMDB00214 & 0.087 & 0.168 & 0.727 & $\downarrow$ \\
\hline 2-Aminoadipic acid & HMDB00510 & 0.093 & 0.168 & 0.732 & $\downarrow$ \\
\hline Phenylalanine & HMDB00159 & 0.094 & 0.168 & 0.755 & $\downarrow$ \\
\hline Homoserine & HMDB00719 & 0.103 & 0.178 & 0.852 & - \\
\hline Methylcysteine & HMDB02108 & 0.114 & 0.187 & 1.330 & - \\
\hline Sarcosine & HMDB00271 & 0.119 & 0.187 & 0.860 & - \\
\hline Arginine & HMDB00517 & 0.120 & 0.187 & 0.866 & - \\
\hline Tyrosine & HMDB00158 & 0.154 & 0.231 & 0.723 & - \\
\hline $\begin{array}{l}\text { Alpha- } \\
\text { aminobutyric acid }\end{array}$ & HMDB00452 & 0.165 & 0.239 & 0.765 & - \\
\hline Kynurenine & HMDB00684 & 0.270 & 0.379 & 0.861 & - \\
\hline Glycine & HMDB00123 & 0.335 & 0.439 & 0.902 & - \\
\hline Beta-alanine & HMDB00056 & 0.338 & 0.439 & 0.809 & - \\
\hline Putrescine & HMDB01414 & 0.341 & 0.439 & 0.777 & - \\
\hline Norepinephrine & HMDB00216 & 0.371 & 0.463 & 0.660 & - \\
\hline Glutamic acid & HMDB00148 & 0.399 & 0.485 & 0.850 & - \\
\hline 5-Hydroxylysine & HMDB00450 & 0.417 & 0.494 & 0.630 & - \\
\hline Glutathione & HMDB00125 & 0.497 & 0.573 & 0.841 & - \\
\hline 4-Hydroxyproline & HMDB06055 & 0.565 & 0.635 & 0.934 & - \\
\hline Aspartic acid & HMDB00191 & 0.713 & 0.781 & 0.960 & - \\
\hline Serotonin & HMDB00259 & 0.729 & 0.781 & 0.699 & - \\
\hline Spermidine & HMDB01257 & 0.785 & 0.812 & 0.933 & - \\
\hline O-Phosphoethanolamine & HMDB00224 & 0.794 & 0.812 & 1.015 & - \\
\hline Ethanolamine & HMDB00149 & 0.963 & 0.963 & 0.858 & - \\
\hline
\end{tabular}

Table 1 Summary of the 45 amine metabolites detected in CIA and control mice

CIA, collagen-induced arthritis; Ctrl, control; HMDB, Human Metabolome Database; FC, fold change; FDR, false discovery rate. a: P-values with bold font indicate significant changes between two groups $(P<0.05)$. 


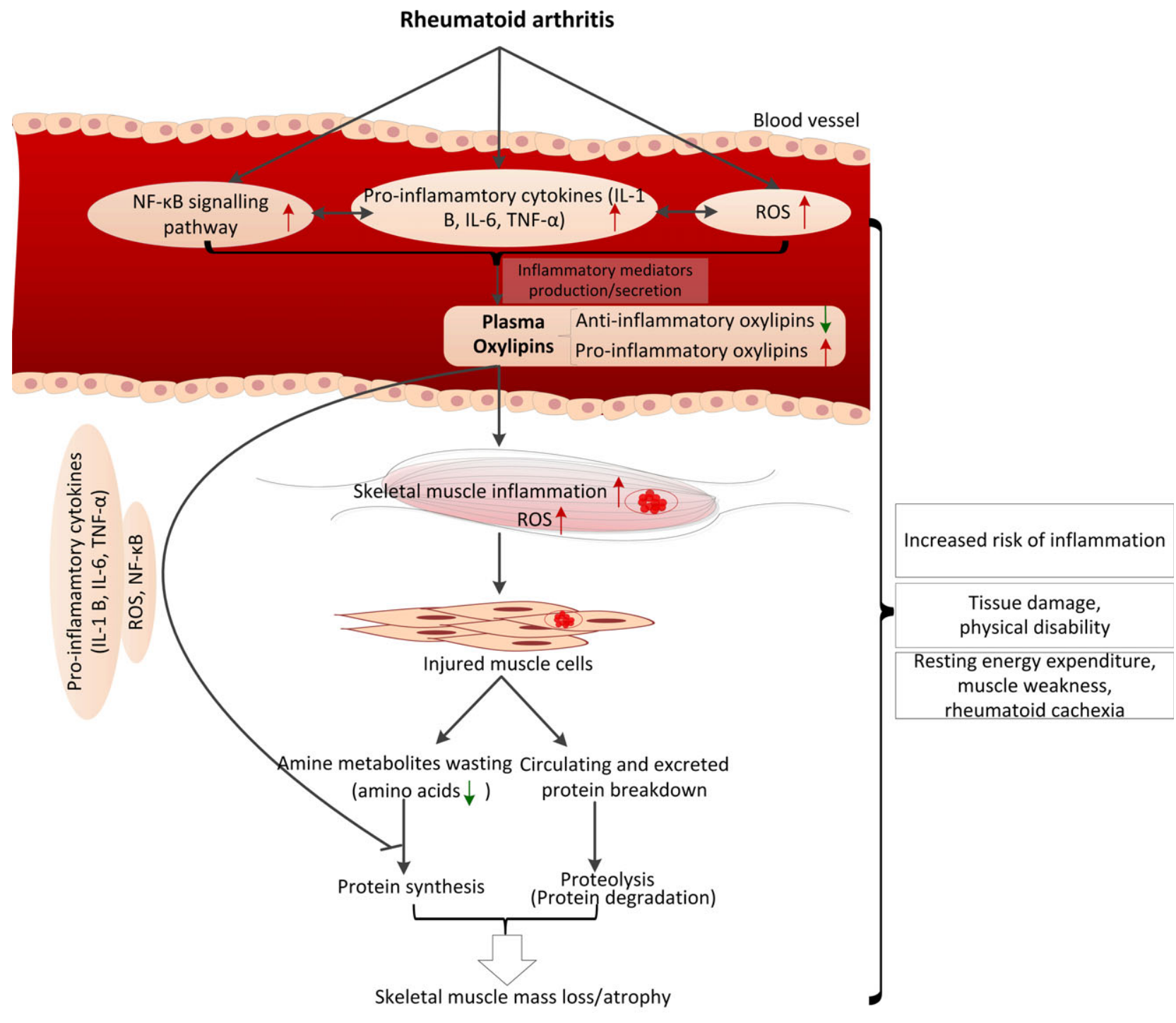

Sequential molecular events in RA

Physiological events in RA

Figure 3 Proposed biological interpretation of muscle wasting in rheumatoid arthritis (RA). In RA, increased levels of inflammatory cytokines, reactive oxygen species (ROS), and nuclear factor (NF)- $\kappa$ B activation play a role in the production of inflammatory oxylipins, which then trigger an inflammatory response in muscle cells. The inflammatory response then increases resting energy expenditure and thermogenesis, leading to amino acid wasting and accelerating protein breakdown. Thereafter, the accelerated protein catabolism and the subsequent reduction in amines, accompanied by the excretion of nitrogen in the urine, causes the muscle mass loss/atrophy that manifests clinically as muscle weakness/cachexia in RA patients.

identify a metabolic 'signature' in the plasma of CIA mice.

Building on the previous report of increased inflammatory mediators and increased ROS-generated oxylipins in CIA mice, ${ }^{29}$ we used a targeted amine platform to evaluate the changes in plasma amine metabolites in age- and gender-matched CIA mice compared with control mice. We found that the plasma amine metabolomes were clearly distinguishable between CIA mice and control mice. Specifically, 20 amine metabolites were significantly lower in the plasma of CIA mice.

Given that certain free amino acids such as branchedchained amino acids are closely associated with protein degradation, amino acids, and their biogenic amines, 
might be used as biomarkers of muscle wasting. ${ }^{50}$ In support of this notion, decreased plasma levels of some amine metabolites have been reported in other diseases (e.g., chronic obstructive pulmonary disease) and have been linked to resting energy expenditure and muscle wasting. ${ }^{51}$ Increased excretion of nitrogen into the urine due to muscle wasting has been reported in RA patients, ${ }^{17,52}$ and increased levels of acyl-carnitines in the urine of RA patients reflect muscle breakdown. ${ }^{53}$ Together, these lines of evidence suggest that muscle wasting is a highly relevant phenomenon related to RA. However, to date relatively few clinical studies have examined muscle wasting in RA by measuring amine metabolite levels. The large decrease in plasma amine metabolite levels (e.g., histidine, valine, leucine, phenylalanine and tryptophan metabolites) is consistent with a previous study of CIA rats by Zhang et al. ${ }^{54}$ The earliest studies of amino acids regulation in RA patients date back to the mid-20th century, ${ }^{55}$ when researchers found decreased levels of several amino acids but were relatively limited with respect to the biological interpretation. Kobayashi et al. measured a similar decrease in some amine metabolites in the plasma of Japanese patients with RA; ${ }^{56}$ although the authors used these results to demonstrate a relationship between ornithine metabolism and inflammation, they did not discuss the possible biological interpretation of non-significantly changed amine metabolites, including alanine, isoleucine, leucine, lysine, serine and valine ${ }^{56}$ In addition, other clinical studies have reported inconsistent changes in the levels of amine metabolites, and did not attribute these changes to muscle wasting. ${ }^{57-61}$ The difference between our CIA mouse model and RA patients with respect to changes in amine metabolites may be due to differences in catabolic processes between mice and humans. Alternatively, the relative complexity of clinical data in patients may mask certain changes in amine metabolites, as various confounding variables are not always taken into consideration in clinical studies, including factors such as age, gender, illness stage, treatment protocol and diet. Our findings indicate that CIA mice are a valuable tool for studying the pathological processes that underlie RA; specifically, this model is easy to induce, and researchers can easily control/exclude confounding factors that may affect the study results, including age, gender, genetic background and drug exposure.

In summary, combining our previous oxylipin results and our current amine metabolomics results allows us to speculate upon the biological relationship between muscle wasting and the inflammatory response in RA (Fig. 3). In addition, our results indicate that muscle wasting conditions such as cachexia can be measured using a metabolomics approach (e.g., by measuring amine metabolites). Lastly, our results indicate that changes in branched-chain amino acids as well as other amine metabolites may reflect muscle wasting status in RA.

\section{CONCLUSIONS}

In summary, using metabolomics, we found that the levels of amine metabolites are systematically decreased in the plasma of CIA mice, which is consistent with similarities between our CIA mouse model and RA patients at the metabolomics level. This result indicates that the muscle wasting and energy expenditure issues (e.g., cachexia) associated with RA, and models of RA, are highly complex.

The cachexia and sarcopenia associated with muscle atrophy, protein breakdown and energy expenditure are not unique to RA. For example, several other chronic inflammatory diseases have been associated with catabolic wasting, including cancer, ${ }^{62}$ human immunodeficiency virus/acquired immunodeficiency syndrome), ${ }^{63}$ type 2 diabetes, ${ }^{64}$ renal failure, uremia $^{65}$ and heart failure. ${ }^{66}$ We therefore hypothesize that systemic decreases in the levels of amine metabolites may reflect muscle mass loss and protein degradation due to inflammation.

Considering the complexity and consequences of muscle wasting in a wide variety of chronic diseases, using a metabolomics-based approach may provide a clearer understanding of the biological processes involved in these diseases.

\section{ACKNOWLEDGEMENTS}

Min He was supported financially by the Chinese Scholarship Council (Scholarship File Number 20108220166). The authors thank Sabine Bos for performing the metabolomics analysis and Prof. Masaki Kobayashi at the Tohoku Institute of Technology, Sendai, Japan for providing laboratory facilities for collecting the plasma samples.

\section{CONFLICT OF INTEREST}

We have no conflict of interest to declare.

\section{ETHICAL APPROVAL}

The study was performed in accordance with the guidelines established in the Guide for the Care and Use of 
Laboratory Animals published by the National Institutes of Health. All experiments were approved by the Tohoku Institute of Technology Research Ethics Committee, Sendai, Japan (approval date: January 18, 2009).

\section{REFERENCES}

1 Alamanos Y, Drosos A (2005) Epidemiology of adult rheumatoid arthritis. Autoimmun Rev 4, 130-6.

2 Gabriel SE (2001) The epidemiology of rheumatoid arthritis. Rheum Dis Clin North Am 27, 269-81.

3 Tobón GJ, Youinou P, Saraux A (2010) The environment, geo-epidemiology, and autoimmune disease: Rheumatoid arthritis. J Autoimmun 35, 10-14.

4 Choy EH, Panayi GS (2001) Cytokine pathways and joint inflammation in rheumatoid arthritis. N Engl J Med 344, 907-16.

5 Firestein GS (2003) Evolving concepts of rheumatoid arthritis. Nature 423, 356-61.

6 Liu H (2003) The role of apoptosis in rheumatoid arthritis. Curr Opin Pharmacol 3, 317-22.

7 Han Z, Boyle DL, Manning AM, Firestein GS (1998) AP-1 and NF-kB regulation in rheumatoid arthritis and murine collagen-induced arthritis. Autoimmunity 28, 197-208.

8 Makarov SS (2001) NF-kappa B in rheumatoid arthritis: a pivotal regulator of inflammation, hyperplasia, and tissue destruction. Arthritis Res 3, 200-6.

9 Ottonello L, Cutolo M, Frumento G et al. (2002) Synovial fluid from patients with rheumatoid arthritis inhibits neutrophil apoptosis: role of adenosine and proinflammatory cytokines. Rheumatology (Oxford) 41, 1249-60.

10 Ottonello L, Frumento G, Arduino N et al. (2002) Delayed neutrophil apoptosis induced by synovial fluid in rheumatoid arthritis: role of cytokines, estrogens, and adenosine. Ann N Y Acad Sci 966, 226-31.

11 Bartok B, Firestein GS (2010) Fibroblast-like synoviocytes: key effector cells in rheumatoid arthritis. Immunol Rev 233, 233-55.

12 Yanaba K, Hamaguchi Y, Venturi GM et al. (2007) B Cell Depletion Delays Collagen-Induced Arthritis in Mice: Arthritis Induction Requires Synergy between Humoral and Cell-Mediated Immunity. J Immunol 179, 1369-80.

13 Raza K, Scheel-Toellner D, Lee C-Y et al. (2006) Synovial fluid leukocyte apoptosis is inhibited in patients with very early rheumatoid arthritis. Arthritis Res Ther 8, R120.

14 Cascão R, Rosário HS, Souto-Carneiro MM, Fonseca JE (2010) Neutrophils in rheumatoid arthritis: More than simple final effectors. Autoimmun Rev 9, 531-5.

15 Walsmith J, Roubenoff R (2002) Cachexia in rheumatoid arthritis. Int J Cardiol 85, 89-99.

16 Roubenoff R (2000) Sarcopenic obesity: does muscle loss cause fat gain? Lessons from rheumatoid arthritis and osteoarthritis. Ann N Y Acad Sci 904, 553-7.
17 da Rocha OM, Batista ADAP, Maestá N et al. (2009) Sarcopenia in rheumatoid cachexia: Definition, mechanisms, clinical consequences and potential therapies. Rev Bras Reumatol 49, 294-301.

18 Summers GD, Deighton CM, Rennie MJ, Booth AH (2008) Rheumatoid cachexia: a clinical perspective. Rheumatology 47, 1124-31.

19 Roubenoff R, Roubenoff RA, Cannon JG et al. (1994) Rheumatoid cachexia: Cytokine-driven hypermetabolism accompanying reduced body cell mass in chronic inflammation. J Clin Invest 93, 2379-86.

20 Holmdahl R, Bockermann R, Bäcklund J, Yamada H (2002) The molecular pathogenesis of collagen-induced arthritis in mice-a model for rheumatoid arthritis. Ageing Res Rev 1, 135-47.

21 Lindqvist A-KB, Bockermann R, Johansson ÅCM et al. (2002) Mouse models for rheumatoid arthritis. Trends Genet 18, S7-13.

22 Nandakumar KS, Holmdahl R (2005) Efficient promotion of collagen antibody induced arthritis (CAIA) using four monoclonal antibodies specific for the major epitopes recognized in both collagen induced arthritis and rheumatoid arthritis. J Immunol Methods 304, 126-36.

23 Brand DD, Latham KA, Rosloniec EF (2007) Collageninduced arthritis. Nat Protoc 2, 1269-75.

24 Ferraccioli G, Bracci-Laudiero L, Alivernini S et al. (2010) Interleukin- $1 \beta$ and interleukin- 6 in arthritis animal models: roles in the early phase of transition from acute to chronic inflammation and relevance for human rheumatoid arthritis. Mol Med 16, 552-7.

25 Horai N, Nagaoka T, Higuchi I et al. (2013) Muscle wasting associated with pathologic change is a risk factor for the exacerbation of joint swelling in collagen-induced arthritis in cynomolgus monkeys. BMC Musculoskelet Disord 14, 205.

26 Hartog A, Hulsman J, Garssen J (2009) Locomotion and muscle mass measures in a murine model of collageninduced arthritis. BMC Musculoskelet Disord 10, 59.

27 Ramautar R, Berger R, van der Greef J, Hankemeier T (2013) Human metabolomics: Strategies to understand biology. Curr Opin Chem Biol 17, 841-6.

28 Van Wietmarschen H, Van Der Greef J (2012) Metabolite space of rheumatoid arthritis. Br J Med Med Res 2, 469-83.

29 He M, Van Wijk E, Berger R et al. (2015) Collagen induced arthritis in DBA / $1 \mathrm{~J}$ mice associates with oxylipin changes in Plasma. Mediators Inflamm 2015, 1-11.

30 Yoshino S, Sasatomi E, Ohsawa M (2000) Bacterial lipopolysaccharide acts as an adjuvant to induce autoimmune arthritis in mice. Immunology 99, 607-14.

31 van Wijk E, Kobayashi M, van Wijk R, van der Greef J (2013) Imaging of ultra-weak photon emission in a rheumatoid arthritis mouse model. PLoS ONE 8, e84579.

32 Noga MJ, Dane A, Shi S et al. (2012) Metabolomics of cerebrospinal fluid reveals changes in the central nervous 
system metabolism in a rat model of multiple sclerosis. Metabolomics 8, 253-63.

33 Xia J, Sinelnikov I V, Han B, Wishart DS (2015) MetaboAnalyst 3.0-making metabolomics more meaningful. Nucleic Acids Res 43, 251-7.

34 Hitchon CA, El-Gabalawy HS (2004) Oxidation in rheumatoid arthritis. Arthritis Res Ther 6, 265-78.

35 Ozkan Y, Yardým-Akaydýn S, Sepici A et al. (2007) Oxidative status in rheumatoid arthritis. Clin Rheumatol 26, 64-8.

36 Simmonds RE, Foxwell BM (2008) Signalling, inflammation and arthritis: NF- $\kappa \mathrm{B}$ and its relevance to arthritis and inflammation. Rheumatology 47, 584-90.

37 Kapoor M, Kojima F, Crofford LJ (2006) Arachidonic acidderived eicosanoids in rheumatoid arthritis: implications and future targets. Fut Rheumatol 1, 323-30.

38 Peterson JM, Feeback KD, Baas JH, Pizza FX (2006) Tumor necrosis factor-alpha promotes the accumulation of neutrophils and macrophages in skeletal muscle. J Appl Physiol 101, 1394-9.

39 Madihally SV, Toner M, Yarmush ML, Mitchell RN (2002) Interferon gamma modulates trauma-induced muscle wasting and immune dysfunction. Ann Surg 236, 649-57.

40 Nagaraju K, Raben N, Merritt G et al. (1998) A variety of cytokines and immunologically relevant surface molecules are expressed by normal human skeletal muscle cells under proinflammatory stimuli. Clin Exp Immunol 113, 407-14.

41 Connor AM, Mahomed N, Gandhi R et al. (2012) TNF $\alpha$ modulates protein degradation pathways in rheumatoid arthritis synovial fibroblasts. Arthritis Res Ther 14, R62.

42 Rall LC (2004) Rheumatoid cachexia: metabolic abnormalities, mechanisms and interventions. Rheumatology 43, 1219-23.

43 Masuko K (2014) Rheumatoid cachexia revisited: a metabolic co-morbidity in rheumatoid arthritis. Front Nutr 1, 1-7.

44 Guttridge DC, Mayo MW, Madrid LV et al. (2000) NF-kappaB-induced loss of MyoD messenger RNA: possible role in muscle decay and cachexia. Science 289, 2363-6.

45 Eguchi J, Koshino T, Takagi T et al. (2002) NF-kappa B and I-kappa B overexpression in articular chondrocytes with progression of type II collagen-induced arthritis in DBA/1 mouse knees. Clin Exp Rheumatol 20, 647-52.

46 Cai D, Frantz JD, Tawa NE et al. (2004) IKK $\beta / N F-\kappa B$ activation causes severe muscle wasting in mice. Cell 119, 285-98.

47 Ling PR, Schwartz JH, Bistrian BR (1997) Mechanisms of host wasting induced by administration of cytokines in rats. Am J Physiol 272, E333-9.

48 Barbieri E, Sestili P (2012) Reactive Oxygen Species in Skeletal Muscle Signaling. J Signal Transduct 2012, 1-17.

49 Barreiro E, De La Puente B, Busquets S et al. (2005) Both oxidative and nitrosative stress are associated with muscle wasting in tumour-bearing rats. FEBS Lett 579, 1646-52.

50 Nicastro H, Da Luz CR, Chaves DFS et al. (2012) Does branched-chain amino acids supplementation modulate skeletal muscle remodeling through inflammation modulation? Possible mechanisms of action J Nutr Metab 2012, $1-10$.

51 Pouw EM, Schols AM, Deutz NE, Wouters EF (1998) Plasma and muscle amino acid levels in relation to resting energy expenditure and inflammation in stable chronic obstructive pulmonary disease. Am J Respir Crit Care Med 158, 797-801.

52 Kotler DP (2000) Cachexia. Ann Intern Med 133, 622-34.

53 van Wietmarschen HA, Dai W, van der Kooij AJ et al. (2012) Characterization of rheumatoid arthritis subtypes using symptom profiles, clinical chemistry and metabolomics measurements. PLoS ONE 7, e44331

54 Zhang H, Fu P, Ke B et al. (2014) Metabolomic analysis of biochemical changes in the plasma and urine of collageninduced arthritis in rats after treatment with Huang-LianJie-Du-Tang. J Ethnopharmacol 154, 55-64.

55 Nettelbladt E (1959) On the metabolism of amino acids and proteins in rheumatoid arthritis. Acta Rheumatol Scand 5, 49-52.

56 Kobayashi T, Okada M, Ito S et al. (2014) Amino acid profiles in relation to chronic periodontitis and rheumatoid arthritis. Open J Stomatol 2014, 49-55.

57 Adams SB, Setton LA, Kensicki E et al. (2012) Global metabolic profiling of human osteoarthritic synovium. Osteoarthritis Cartilage 20, 64-7.

58 Madsen RK, Lundstedt T, Gabrielsson J et al. (2011) Diagnostic properties of metabolic perturbations in rheumatoid arthritis. Arthritis Res Ther 13, R19.

59 Shet K, Siddiqui SM, Yoshihara H et al. (2012) High-resolution magic angle spinning NMR spectroscopy of human osteoarthritic cartilage. NMR Biomed 25, 538-44.

60 Kim S, Hwang J, Xuan J et al. (2014) Global metabolite profiling of synovial fluid for the specific diagnosis of rheumatoid arthritis from other inflammatory arthritis. PLOS ONE 9, 1-9.

61 Zhang Q, Li H, Zhang Z et al. (2015) Serum metabolites as potential biomarkers for diagnosis of knee osteoarthritis. Dis Markers 2015, 1-7.

62 O'Connell TM, Ardeshirpour F, Asher SA et al. (2008) Metabolomic analysis of cancer cachexia reveals distinct lipid and glucose alterations. Metabolomics 4, 216-25.

63 Grunfeld C, Feingold KR (1994) Metabolic disturbances and wasting in the acquired immunodeficiency syndrome. N Engl J Med 330, 1041-6.

64 Park S, Goodpaster B, Lee J et al. (2009) Excessive loss of skeletal muscle mass in older adults with type 2 diabetes. Diabetes Care 32, 1993-7.

65 May RC, Kelly RA, Mitch WE (1987) Mechanisms for defects in muscle protein metabolism in rats with chronic uremia. Influence of metabolic acidosis. J Clin Invest 79, 1099-103.

66 Anker SD, Coats AJS (1999) Cardiac cachexia: a syndrome with impaired survival and immune and neuroendocrine activation. Chest 115, 836-47. 